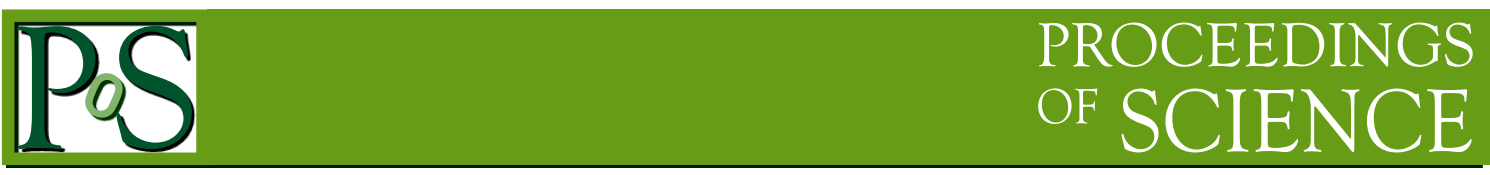

\title{
Heavy ion beam test at CERN-SPS with the CALET Structure Thermal Model
}

\author{
Tadahisa TAMURA for the CALET collaboration \\ Kanagawa University \\ 3-27-1 Rokkakubashi, Kanagawa-ku, Yokohama, Kanagawa, Japan \\ E-mail: ttamura@kanagawa-u.ac.jp
}

We will report testing and calibration of the heavy-ion energy and charge resolution of the CALET cosmic-ray instrument that will fly on the International Space Station in 2015. CALET will measure the energy spectra and arrival directions of cosmic-ray electrons to $20 \mathrm{TeV}$ and hadrons to $1 \mathrm{PeV}$ with exceptional resolution. It will measure the spectra of high-energy nuclei to about $Z=40$. It will also measure the cosmic gamma radiation with superior resolution to search for signatures of dark matter annihilation in the gamma-ray and electron spectra. We performed beam tests at CERN-SPS in February and March 2015 to calibrate energy, angular and charge resolution with direct primary beams and secondary fragments of Ar of 13, 19, and $150 \mathrm{~A} \mathrm{GeV/c}$. The beam tests were carried out using a test instrument that is functionally equivalent to the calorimeter (CAL) of CALET. The purpose of our ion run, experimental method and setups, and preliminary results are reported.

The 34th International Cosmic Ray Conference

30 July- 6 August, 2015

The Hague, The Netherlands 


\section{Introduction}

In order to observe high energy electrons, gamma-rays, and nuclei on the International Space Station, we have developed the CALET (CALorimetric Electron Telescope) instrument. CALET was approved by the Japanese Aerospace Exploration Agency (JAXA) in March 2010. It has been installed on the H-II Transfer Vehicle 5 (HTV5). The H-IIB Launch Vehicle No.5 (H-IIB F5) with the HTV5 will be launched at the Tanegashima Space Center (TNSC) in August 2015. CALET will start nominal observations after 90 days initial phase including check-out on orbit [1].

The primary target is the observation of electrons in the high energy region up to $20 \mathrm{TeV}$. Supernova remnants (SNRs) are anticipated as a candidate source which can accelerate electrons up to such high energies. While a simple power law spectrum of electrons below $1 \mathrm{TeV}$ can result from integration of all SNRs in the Galaxy, we expect that characteristic features in the energy spectrum of electrons can be detected in $\mathrm{TeV}$ region. That is because there exist only a few candidate objects to provide such high energy electrons within $1 \mathrm{kpc}$ from the Earth. If we detect some bump structures in the electron energy spectrum and anisotropy in arrival directions of electrons over $1 \mathrm{TeV}$, we can infer that they result from nearby SNR and it will become an important evidence that SNRs are really the source of high energy electrons. CALET has very good energy resolution of less than about $3 \%$ above $100 \mathrm{GeV}$ which is important to detect structures in the energy spectrum, and also possesses a high rejection power of about $10^{5}$ against background protons. Such high rejection power is indispensable because the ratio of background protons to electrons becomes larger at the higher energies. CALET's thick calorimeter (30 r.l.) is effective to achieve not only good energy resolution but also high background rejection power. Such capabilities are also very important to datect a possible enhancement in the electron flux over $100 \mathrm{GeV}$.

CALET will have the capability to measure the proton spectrum up to $900 \mathrm{TeV}$, helium up to $400 \mathrm{TeV} / \mathrm{n}$, carbon and silicon up to $20 \mathrm{TeV} / \mathrm{n}$, and iron up to $10 \mathrm{TeV} / \mathrm{n}$ in a 5 year mission. We can inspect the hardening of the energy spectrum of protons and heliums at around $200 \mathrm{GeV} / \mathrm{n}$ and the difference in their power indices. If we detect a cutoff in the proton spectrum in the 100 $\mathrm{TeV}$ region, we can verify the shock acceleration. By observing the dependence of cosmic-ray composition on energy, we can study the acceleration and propagation mechanism of cosmic rays. CALET can observe the ratio of the primary to the secondary cosmic rays, for example boron to carbon above $1 \mathrm{TeV} / \mathrm{n}$, with sufficiently high statistics in 5 years of observation to investigate the energy dependence of the diffusion constant. It will distinguish models of magnetic fields in the Galaxy.

\section{Instrumentation}

The CALET instrument will be installed to the 9th attachment point of the Japanese Experiment Module Exposed Facility (JEM-EF). It is assembled into the standard payload as shown in Figure 1. The main instrument of CALET is called CAL which makes up the front part of the payload and consists of three detector components (Figures 2, 3).

A charge detector (CHD) attached at the top as the first detector component is composed of plastic scintillator paddles assembled in two layers with a surface area of $450 \times 450 \mathrm{~mm}^{2}$. Each layer has 14 plastic scintillator paddles of $450(\mathrm{~L}) \times 32(\mathrm{~W}) \times 10(\mathrm{H}) \mathrm{mm}^{3}$. The two layers are assembled in orthogonal directions to each other. Each paddle is read out with one photo multiplier 
tube (PMT). The CHD can determine charges of incident particles up to $Z=40$ with a charge resolution of 0.15 e for $\mathrm{B}$ and $\mathrm{C}$ and about $0.3 \mathrm{e}$ for $\mathrm{Fe}$.

An imaging calorimeter (IMC) at the middle of the CAL is the second detector. It takes fine shower images at the beginning of the shower development with $1 \mathrm{~mm}$ square scintillating fibers. 448 scintillating fibers are glued side by side in parallel on an aluminum honeycomb substrate to form a scintillating fiber belt. Two scintillating fiber belts in orthogonal direction to each other make up one layer. Tungsten plates as convertors are interleaved between the scintillating fiber layers. Thickness of the upper 5 tungsten plates are $0.7 \mathrm{~mm}$ of 0.2 radiation length (r.1.) and that of the lower 2 plates are $3.5 \mathrm{~mm}$ of $1 \mathrm{r}$.1. By the tungsten converter, incident particles start shower development in the IMC. The shower images are taken with the scintillating fibers. The IMC consists of 8 layers and the number of scintillating fibers amounts to 7168 channels. Each scintillating fiber is read out by one anode of a 64 channel multi-anode PMT (MaPMT). We use analog ASIC chips of VA32HDR14.3 to read out pulse signals from the 112 MaPMTs. The front end circuit (FEC) achieves high dynamic range of 3000. The imaging capability of the IMC enables mainly to determine the incident particle directions and starting points of shower development.

A total absorption calorimeter (TASC) is located at the bottom of the CAL as the third detector component to determine energies of incident particles with a very high precision. It has a hodoscope type structure composed of lead tungstate (PWO) scintillation crystals. The dimensions of each PWO are $20(\mathrm{H}) \times 19$ (W) $\times 326$ (L) $\mathrm{mm}^{3}$. The TASC has 12 layers with 16 PWOs in each layer. The PWOs in each layer are arranged at 90 degrees with respect to the previous layer. An avalanche photodiode (APD) and photodiode (PD) are attached to each PWO crystal as a photo sensor to read out the scintillation pulses. Sensitive range of the APD and the PD is different by 3 orders of magnitude. That is because the APD has a higher gain by about 50 times and a larger photo sensitive area by about 20 times than the PD. The readout electronics for the APD/PD has two gain systems different by about 30 times. The readout of the PWO by the $\mathrm{APD} / \mathrm{PD}$ has a high dynamic range of $10^{7}$ in total. The total thickness of the TASC is 27 r.l., making it possible to measure incident particles up to high energies with very good energy resolution. The TASC's imaging capability makes it a powerful tool to identify electrons and reduce proton backgrounds by measuring the shower longitudinal development and lateral spread. At the highest CALET energies, electrons will be contained in the TASC calorimeter and have narrower lateral spreads than protons.

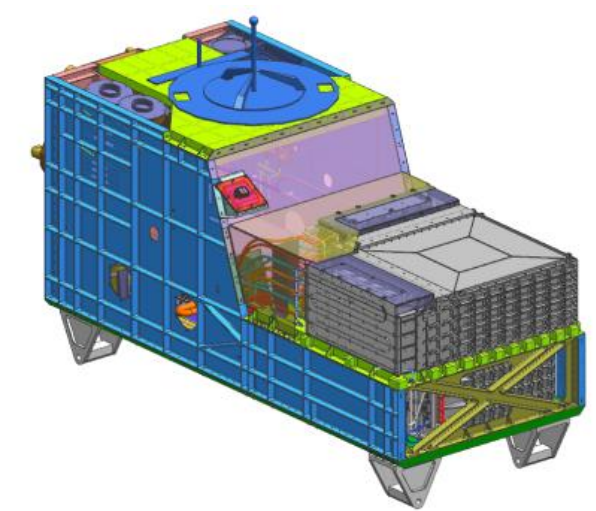

Fig.1: The CALET instrument is assembled into the standard payload for the JEM.

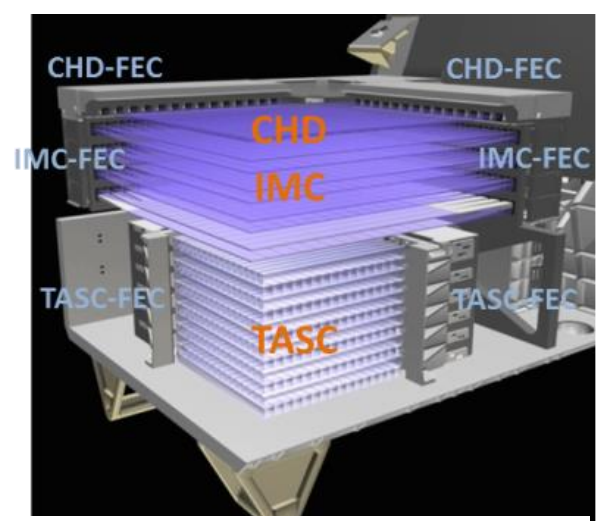

Fig. 2: The main detector of CALET which is called CAL is composed of three components. 


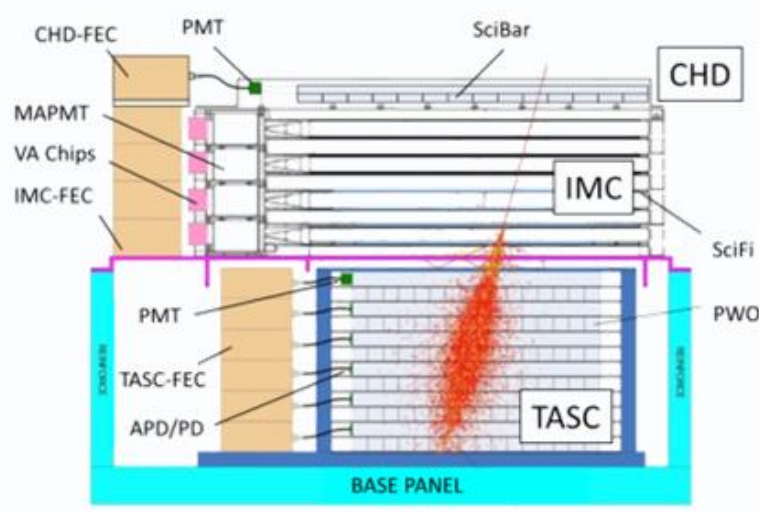

Fig. 3: The CAL consists of CHD, IMC and TASC.

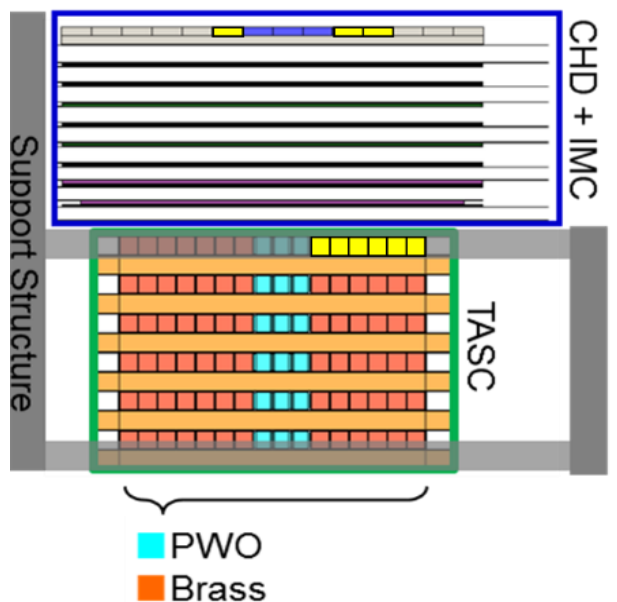

Fig. 4: The beam test instrument reassembled in 2015. Plastic scintillators added in CHD and PWO scintillators added in TASC are indicated by yellow.

\section{Beam tests at CERN-SPS}

A series of CALET beam experiments have been carried out at test lines of the Super Proton Synchrotron (SPS) accelerator at CERN. Prior to formal approval of the CALET project by JAXA, initial beam tests were conducted at CERN-SPS as research and development for the CALET proposal. In particular, BGO and PWO crystals were tested at CERN-SPS in 2009. After CALET was approved and the detector configuration was fixed, the first CALET prototypes were fabricated with one-directional readout and limited channels, and tested with electron and proton beams at CERN-SPS in 2010 and 2011.

A Breadboard Model (BBM) and Structure Thermal Model (STM) were constructed as part of the electronics, detector and support structure development. No full Engineering Model (EM) was constructed due to the strategy to skip the EM and proceed to the Proto Flight Model (PFM) after the BBM and the STM developments. The PFM will be launched as the Flight Model (FM) after all required proto flight tests are completed. In the absence of an EM, we utilized the BBM electronics and the STM detector structures to construct a test instrument for the beam tests which was functionally equivalent to the PFM. These beam tests were used to verify and understand the simulations used to design the CALET instrument and evaluate its performance.

Table 1: Summary of the beam experiments in 2015

\begin{tabular}{|c|c|c|l|}
\hline & \multicolumn{1}{|c|}{ Dates } & $\begin{array}{c}\text { Beam momenta } \\
{[\mathrm{GeV} / \mathrm{c}]}\end{array}$ & Experimental conditions \\
\hline 1 & Feb.11-Feb.15 & $150 \mathrm{~A}$ & Behind UA9 (parasitic): pure Ar \\
\hline 2 & Feb.15-Feb.20 & $150 \mathrm{~A}$ & Main user (w/o Si detector): pure Ar, fragments \\
\hline 3 & Feb.20-Feb.23 & $150 \mathrm{~A}$ & Behind Proba-V (parasitic): pure Ar \\
\hline 4 & Feb.23-Feb.25 & $13 \mathrm{~A}$ & Behind Proba-V (parasitic): pure Ar \\
\hline 5 & Feb.25-Mar.3 & $13 \mathrm{~A}$ & Main user (with Si detetctor): pure Ar, fragments \\
\hline 6 & Mar.3-Mar.17 & $19 \mathrm{~A}$ & Main user (with Si detector): pure Ar, fragments \\
\hline
\end{tabular}




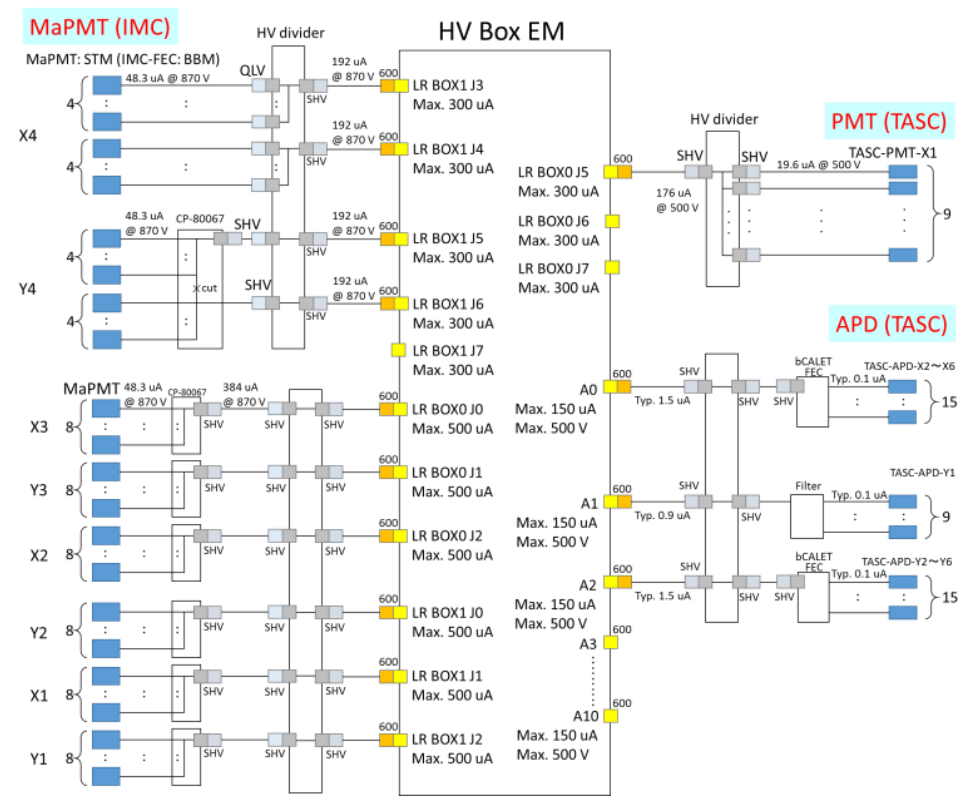

Fig.5 : Block diagram of HV supplyments with the HV Box EM.

Beam tests were carried out with the test instrument at CERN-SPS in 2012 [2]. The 2012 exposures provided data from proton and electron beams only. In 2013, we performed ion runs at CERN-SPS with the BBM electronics before a long shutdown (more than one year) of the accelerator beam.

CERN-SPS beam operations resumed in late 2014. We exposed the test instrument with the BBM electronics and the STM detector structures to Ar beams from February to March 2015. The main goal of the 2015 exposure was to compare simulation results with experimental data, in particular regarding energy resolution for heavy ions at the highest momentum available. An Ar beam at $150 \mathrm{~A} \mathrm{GeV} / \mathrm{c}$ was made available for 5 days as a main user together with beams at $19 \mathrm{~A}$ $\mathrm{GeV} / \mathrm{c}$ and $13 \mathrm{~A} \mathrm{GeV} / \mathrm{c}$. The beam experimental conditions are summarized in Table 1 .

Compared to the earlier exposures, in 2015 we increased the number of CHD scintillators from 6 paddles ( 3 paddles in the $\mathrm{X}$ direction and 3 paddles in the $\mathrm{Y}$ direction) to 12 paddles (6 paddles in $\mathrm{X}$ and 6 paddles in $\mathrm{Y}$ ) as shown in Figure 4. We also increased the number of PWO scintillators from $36 \operatorname{logs}$ to 48 logs. In 2012, 3 PWO scintillators and 13 brass bars as a mass dummy were arranged in one layer. Based on simulation studies, in 2015 we added 6 PWO logs on one side of the first TASC layer and the other 6 logs on one side of the second TASC layer.

A High Voltage (HV) box EM [3] was also available for the 2015 exposures, providing high voltages for the 64 MaPMTs of the IMC, the 9 PMTs of the TASC first layer, and the 39 APDs as shown in Figure 5. All available channels of the HV box EM were checked for linearity of output voltages relative to HV setting values. An example of the linearity check done for one channel with no load on the output is shown in Figure 6. The HV box EM was controlled by a Windows PC. Therefore we could change the high voltages at the beam control room as needed.

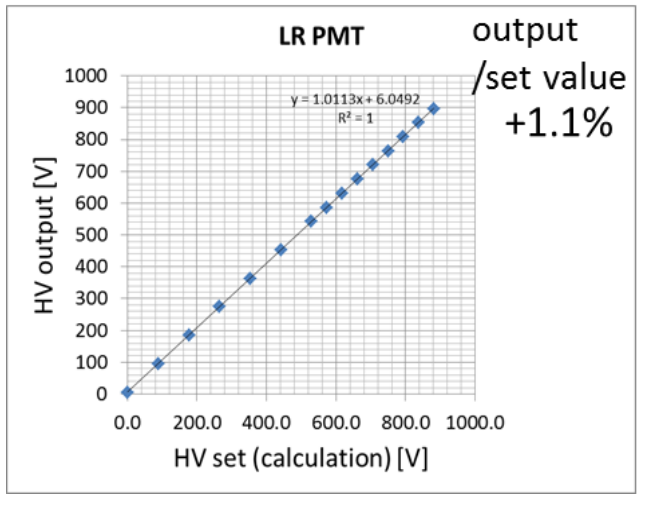

Fig. 6 : An example of a linearity check of output voltages of the HV box EM. 
We carried out the Ar ion runs in the H8 beam line at the SPS Prevessin site (Figure 7). The DAQ system consisted of a VME computer system, a NIM system, and a CAMAC system. We used the VME computer to control trigger sequence and the CAMAC system and to read data from the BBM electronics and VME modules (a charge ADC and a scaler). The CAMAC system contained peak hold ADC modules to read the CHD plastic scintillators and sum of last dynode signals of the MaPMTs. The instrument was mounted on a moving table and covered with black sheets to shield the light. The table position could be translated vertically and horizontally from the beam control room, enabling us to vary beam incident positions without access to the beam area.
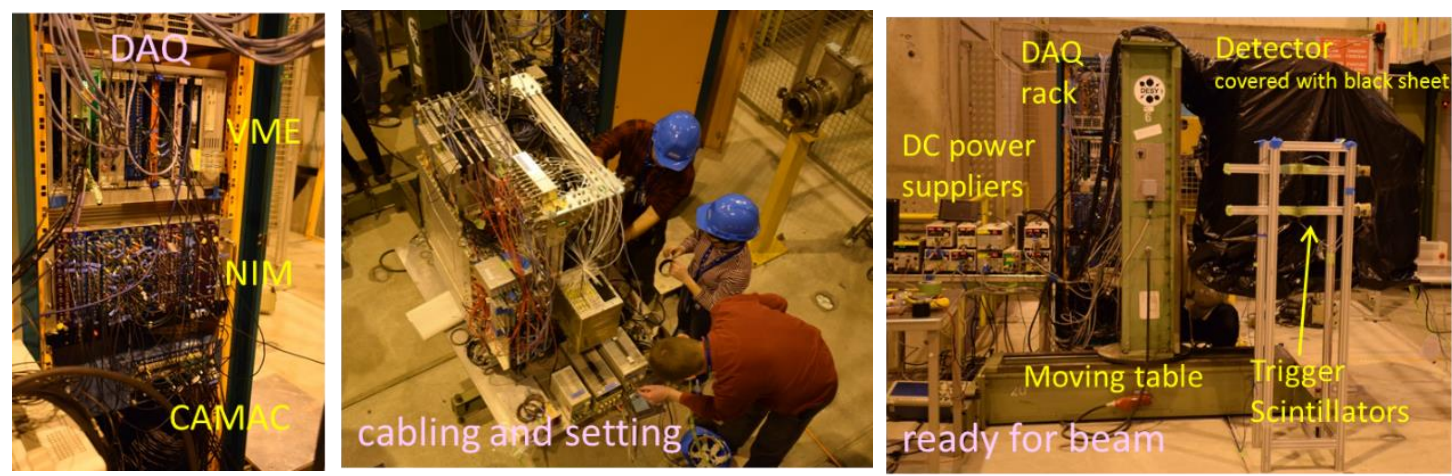

Fig. 7 : Illustration of data aquisition electronics and the beam test instrument at $\mathrm{H} 8$.

The highest beam energy of $150 \mathrm{~A} \mathrm{GeV} / \mathrm{c}$ was available to CALET for 5 days between Feb. 15 and Feb. 20 as a main user. Before and after that we operated parasitically for 7 days in total with UA9 and Proba-V. We also obtained exposures at 13Aand 19A GeV/c. Schematic drawings of the configuration of the beam test instrument in the $\mathrm{H} 8$ beam area are shown in Figure 8. A Si tracker [4] was positioned upstream the instrument for the $13 \mathrm{~A}$ and $19 \mathrm{~A} \mathrm{GeV} / \mathrm{c}$ exposures, making it possible to determine the tracks of the incident particles and their $\mathrm{dE} / \mathrm{dx}$ precisely to distinguish particle charges.
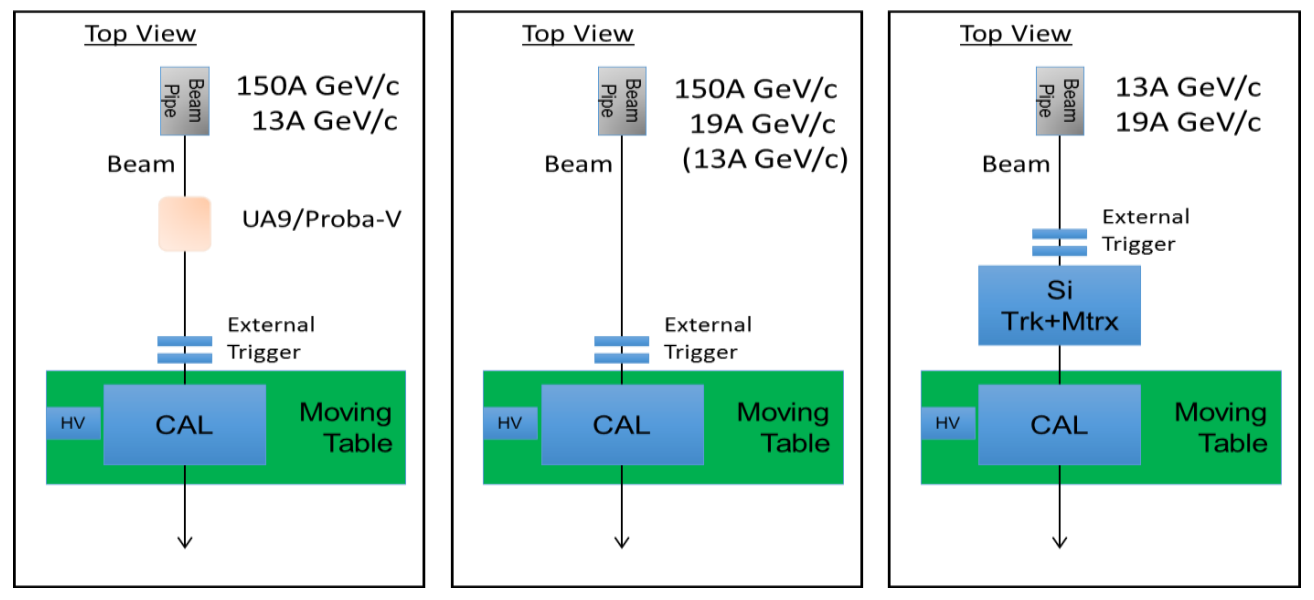

Fig. 8 : Configuration of the beam test instrument, indicated as CAL.

The primary ${ }^{40} \mathrm{Ar}$ beam was used directly and also fragments produced after irradiating a polyethylene target of thickness $40 \mathrm{~mm}$. Fragments with the desired values of $\mathrm{A} / \mathrm{Z}$ were selected with magnets in the beam line: Fragments up to ${ }^{36} \mathrm{Ar}$ were obtained by selecting particles of $\mathrm{A} / \mathrm{Z}$ $=2.0$ and fragments up to ${ }^{40} \mathrm{Ar}$ were obtained by selecting $\mathrm{A} / \mathrm{Z}=2.2$. The ratio of ${ }^{36} \mathrm{Ar}$ to the other 
fragments was reduced in the former case while that of ${ }^{40} \mathrm{Ar}$ was relatively large in the latter case because ${ }^{40} \mathrm{Ar}$ was the primary beam. Figure 9 shows a sample event from a $13 \mathrm{~A} \mathrm{GeV} / \mathrm{c}{ }^{40} \mathrm{Ar}$ primary beam obtained with a quick look event monitor in the beam control room. A total of 7.5 M events were accumulated.

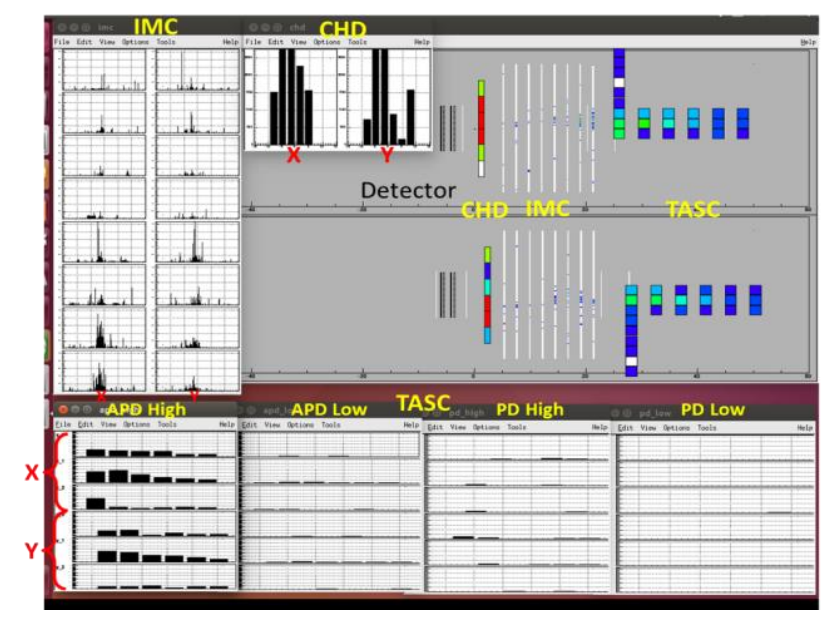

Fig.9 : A sample event from ${ }^{40} \mathrm{Ar}$ primary beam at $13 \mathrm{~A} \mathrm{GeV} / \mathrm{c}$.

A test was performed with two CHD plastic scintillator paddles to study the effects of quenching and delta rays. While signals from the plastic scintillators are reduced for heavy ions by the quenching effect, signals will be increased by the effect of delta rays generated in an aluminum plate support structure and plastic scintillator upstream. Because the effect of the delta rays from upstream materials is just several percent relative to the energy deposit of a minimum ionizing particle (MIP), we tried to confirm that the effect was really caused by the delta rays and not by a difference in the light output performance of each scintillator. We positioned the two CHD plastic scintillator paddles closely together and took data at incident beam angles of 0,15 , 30,45 , and 60 degrees. At an incident beam angle of 0 degrees, we took data by exchanging positions of the paddles, and also took data with a $2 \mathrm{~mm}$ thick aluminum plate set in front.

\section{Results}

Figure 10 shows a preliminary pulse height distribution of the sum of the 2 layers in CHD obtained from fragments selected with $\mathrm{A} / \mathrm{Z}=2.2$ at $150 \mathrm{~A} \mathrm{GeV} / \mathrm{c}$. Peaks due to fragments from Deuteron to Ar are clearly separated. The ${ }^{40} \mathrm{Ar}$ peak at the right is most prominent. On the other hand, light elements with $\mathrm{A} / \mathrm{Z}=2.0$ are suppressed. Figure 11 shows another preliminary result for fragments selected with $\mathrm{A} / \mathrm{Z}=2.0$. Here the light elements are much more dominant while the ${ }^{36} \mathrm{Ar}$ peak is relatively reduced. Further detailed analyses on charge resolutions, energy resolutions for each heavy ion, and so forth are now underway. Results will be shown elsewhere in these proceedings $[5,6]$.

\section{Summary}

We obtained beam test data with electrons, protons, and heavy ions at CERN-SPS with our beam test instrument utilizing the BBM electronics and the STM detectors before CALET was launched. The beam test instrument is functionally equivalent to the CALET FM. However, since the complete set of FM detector elements was not available for the beam tests, detector performance is not identical for the CALET FM and the beam test instrument. Therefore, we 
aimed not at calibrating the detector performance of the CALET FM with the SPS beam tests but at comparing the experimental results with simulations which we have used to design the CALET detectors and to evaluate their performance. We are now analyzing all data taken in the beam tests to obtain angular resolution, energy resolution, proton rejection power, charge resolution, and so on $[5,6,7]$. We will summarize and conclude the series of beam test results shortly. By comparing these results with simulated data, we can verify validity of our simulations and improve their accuracy.

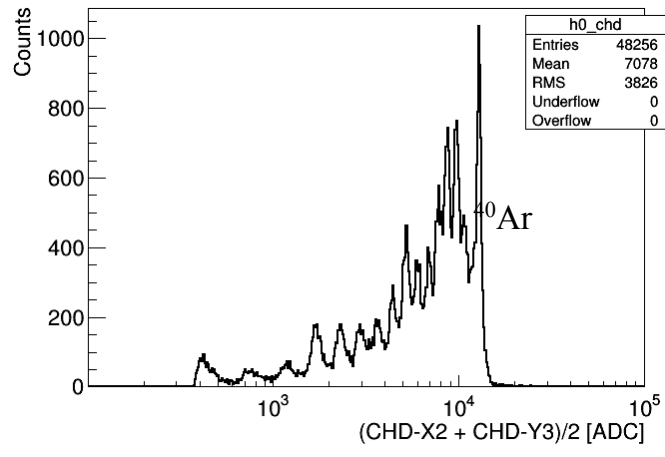

Fig. 10 : Pulse height distribution of sum of the 2 layers in $\mathrm{CHD}$ for fragments $(\mathrm{A} / \mathrm{Z}=2.2)$. HV for $\mathrm{CHD}$ was set at $-400 \mathrm{~V}$.

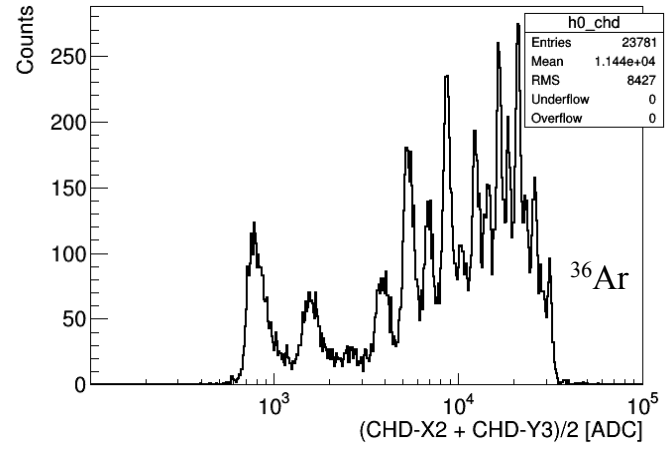

Fig.11 : Pulse height distribution of sum of the 2 layers in $\mathrm{CHD}$ for fragments $(\mathrm{A} / \mathrm{Z}=2.0)$. HV for $\mathrm{CHD}$ was set at $-450 \mathrm{~V}$.

\section{Acknowledgment}

We extend sincere thanks to Dr. Henric Wilkens who is the SPS coordinator, and Dr. Ilias Efthymiopoulos, Dr. Nikolaos Charitonidis, Dr. Michael Jeckel, and other staffs in CERN for their support to our successful beam tests. This work was supported by KAKENHI 25257203.

\section{References}

[1] S. Torii for the CALET Collaboration, "Calorimetric Electron Telescope (CALET) for the International Space Station”, $34^{\text {th }}$ International Cosmic Ray Conference (Hague, The Netherlands) (2015).

[2] T.Tamura for the CALET Collaboration, "Particle beam tests of the Calorimetric Electron Telescope", $33^{\text {th }}$ International Cosmic Ray Conference (Rio de Janeiro, Brazil) (2013).

[3] Beni et al., "Electromagnetic compatibility tests for the space qualification of the high-voltage system of CALET apparatus", Technical Scientific and Research Reports, vol. 5 (2013) 69-165 IFAC special issue.

[4] P.S.Marrocchesi et al., "Beam test performance of a scintillator-based detector for the charge identification of relativistic ions", Nuclear Instruments and Methods in Physics Research $\mathbf{A 6 5 9}$ (2011) 477-483

[5] Paolo Brogi and Pier Simone Marrocchesi for the CALET Collaboration, "CALET measurements with cosmic nuclei: expected performance of tracking and charge identification", $34^{\text {th }}$ International Cosmic Ray Conference (Hague, The Netherlands) (2015).

[6] Y. Akaike for the CALET Collaboration, "CALET energy calibration using CERN-SPS beam tests", $34^{\text {th }}$ International Cosmic Ray Conference (Hague, The Netherlands) (2015).

[7] Gabriele Bigongiari for the CALET Collaboration, "CALET perspectives for calorimetric measurements of high energy electrons based on beam test results", $34^{\text {th }}$ International Cosmic Ray Conference (Hague, The Netherlands) (2015). 